\title{
POLITIQUE, GOUVERNANCE ET ORGANISATION
}

\section{Odile Hologne et al.}

\section{A.D.B.S. | Documentaliste-Sciences de l'Information}

\section{3/2 - Vol. 50}

pages 38 à 45

\section{ISSN 0012-4508}

Article disponible en ligne à l'adresse:

http://www.cairn.info/revue-documentaliste-sciences-de-I-information-2013-2-page-38.htm

Pour citer cet article :

Hologne Odileet al., " Politique, gouvernance et organisation »,

Documentaliste-Sciences de I'Information, 2013/2 Vol. 50, p. 38-45. DOI : 10.3917/docsi.502.0038

Distribution électronique Cairn.info pour A.D.B.S..

(c) A.D.B.S.. Tous droits réservés pour tous pays.

La reproduction ou représentation de cet article, notamment par photocopie, n'est autorisée que dans les limites des conditions générales d'utilisation du site ou, le cas échéant, des conditions générales de la licence souscrite par votre établissement. Toute autre reproduction ou représentation, en tout ou partie, sous quelque forme et de quelque manière que ce soit, est interdite sauf accord préalable et écrit de l'éditeur, en dehors des cas prévus par la législation en vigueur en France. II est précisé que son stockage dans une base de données est également interdit. 
[ p.38 ] Du réseau à l'organisation apprenante : l'animation de la fonction IST

[ p.40 ] Un GIP pour la gouvernance d'Ascodocpsy
[ p.42 ] Les réseaux métiers en IST au CNRS [ p.44] La documentation des Business Schools : mutualisation ou concurrence?

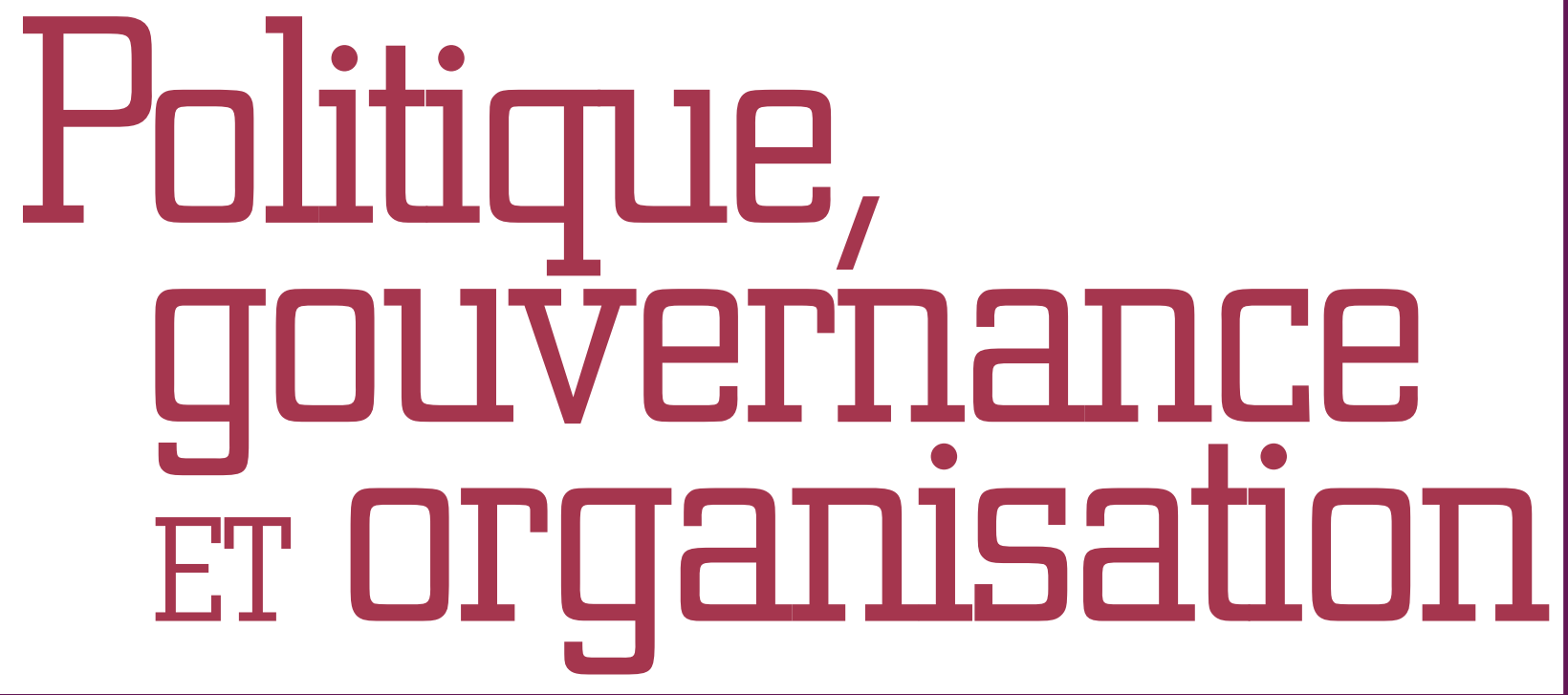

[ évolution ] Des réseaux devenus obsolètes car peu propices à l'animation de projets collectifs et qu'il fallait faire évoluer, voilà une problématique classique. Les pôles imaginés par l'Inra pour favoriser le développement des compétences métiers et l'émergence de nouveaux services représentent de réels instruments de valorisation professionnelle. Le projet FufurIST sera sans nul doute une source d'inspiration.

\section{Du réseau à l'organisation apprenante : l'animation de la fonction IST}

La fonction IST à l'Inra regroupe les métiers des familles documentation et édition de la Branche d'activité professionnelle $\mathrm{F}^{1}$, soit environ 160 personnes pour près de 3500 scientifiques. Ces personnels IST sont disséminés dans différentes structures de recherche ou administratives où ils sont les seuls représentants de leurs compétences. Le pilotage est assuré par une direction déléguée à l'IST au sein de la direction de la valorisation rattachée au directeur général en charge de la recherche.

L'évolution très rapide des technologies de l'information a impacté fortement ces métiers : le chercheur n'a plus besoin du lieu «bibliothèque »ni de la médiation du bibliothécaire, pour accéder à l'information. Ces évolutions influencent l'offre de service de la fonction, les compétences et les modalités d'intervention des professionnels.
Dans un contexte de malaise grandissant, la direction générale a demandé une réflexion sur la gestion prévisionnelle des emplois et compétences de l'IST qui s'est traduite début 2006 par la création du projet FuturIST : futur de la fonction IST. Ce projet de type conduite du changement est décrit dans un article $^{2}$ accessible dans l'archive institutionnelle ProdInra.

\section{Les limites du réseau}

Dans les années 2000, la fonction IST disposait d'une liste de diffusion interne ISTinfo à laquelle tous les personnels IST étaient abonnés. Cette liste, modérée par des animateurs, servait de canal de diffusion d'une information officielle. Les contributions des acteurs du réseau restaient timides. Des réunions annuelles pour faire le point sur les projets rassemblaient les ingénieurs et assistants ingénieurs sans les techniciens (70\% des effectifs). À ce réseau d'échange national se superposaient des réseaux locaux ou des réseaux de départements de recherche animés par 
les responsables IST de ces structures. C'est le dispositif « département » qui était le plus fécond pour l'émergence de projets collectifs tels que les bases de publications (remplacées par ProdInra) ou des dispositifs de veille tels que Thémaclic ${ }^{3}$ du Département SAE2. Le dispositif national d'animation, basé sur un modèle de diffusion d'information top-down tant via la liste de diffusion que les réunions, ne favorisait par l'implication des acteurs potentiels isolés dans leurs structures. Les projets collectifs étaient peu nombreux et les différents réseaux renforçaient le cloisonnement.

\section{Vers une organisation apprenante}

Lors de la dernière étape du projet FuturIST, la nécessité de faire évoluer cette animation est devenue de plus en plus prégnante. Le comité de pilotage a validé la création de pôles de compétences avec une double finalité :

$>$ une finalité Métier : renforcer l'animation et développer des centres de ressources permettant aux professionnels de disposer d'outils et de méthodes partagés, de bénéficier de formations pour enrichir ou diversifier leurs compétences, de rompre l'isolement induit par les multiples rattachements administratifs;

$>$ une finalité Service : contribuer à développer l'offre de services aux scientifiques en s'adaptant aux nouveaux besoins, proposer de nouveaux services tout en veillant à l'équité d'accès sur tous les sites.

L'organisation générale repose sur :

- une équipe noyau dur avec un ou des animateurs et des personnes se consacrant à temps plein ou partiel aux activités du pôle. Elle initie et met en ouvre les actions :

- un comité chargé d'orienter les travaux, de conseiller, de prioriser les actions ;

- les documentalistes qui peuvent être des contributeurs ou des utilisateurs des pôles. S'ils sont des contributeurs, ils ne participent pas à l'activité de plus de deux pôles et déclarent explicitement leur implication ; - des groupes de travail ou des équipes projet composés en fonction des sujets à traiter, à durée de vie limitée ;

- un budget pour la mise en cuvre des projets.

Neuf pôles ${ }^{4}$ ont été créés pour répondre aux besoins des scientifiques identifiés dans le projet FuturIST. Pendant leur conception, les animateurs ont été formés au management. Des actions de communication ont été ciblées tant vers les documentalistes vers leur hiérarchie. Il s'agissait de motiver les documentalistes pour qu'ils s'impliquent dans les pôles et de faire comprendre à la hiérarchie scientifique que cette implication ne faisait pas concurrence avec leur activité courante. Pour les 2 cibles, il fallait souligner qu' "un investissement dans le collectif est nécessaire pour développer les compétences et les outils des professionnels IST. En retour, cet investissement bénéficie à chacun dans ses activités et dans ses projets ».

\section{Forces et faiblesses du dispositif}

Ce dispositif repose sur un fonctionnement non hiérarchique et sa cohésion sur l'énergie que chacun y consacre, proportionnelle aux bénéfices qu'il en retire. Les animateurs dont la mission est définie dans une fiche de fonction générique ont pour rôle principal de faire circuler l'information, de favoriser l'émergence de projets, d'organiser la veille, de gérer les moyens financiers accordés au pôle pour la mise en œuvre des projets arbitrés. La reconnaissance du rôle des animateurs était une question difficile puisque leur entretien annuel est réalisé par un responsable hiérarchique qui ne connait pas l'IST (un directeur d'unité de recherche ou de service administratif). La prise en compte du rôle d'animateur par la commission d'évaluation des ingénieurs a été importante pour valoriser cette activité dans leur progression de carrière.

La fonction IST est devenue une organisation apprenante ${ }^{5}$ selon les 5 critères de Garvin ${ }^{6}$ car on peut avec ce dispositif de pôles :

1. résoudre les problèmes en groupe ;

2. expérimenter via des projets ou des preuves de concept ;

3. tirer les leçons des expériences, des succès comme des échecs;

4. apprendre avec les autres ;

5. transférer les connaissances en les formalisant et les diffusant.

Ingénieur en chef des Ponts, des eaux et forêts, spécialisée en économie,

Odlle Hologne occupe depuis 20 ans des postes liés à l'informatique et à la

valorisation de l'information dans des organismes publics sous tutelle du

ministère de l'Agriculture. Directrice déléguée à l'IST de l'Inra, depuis 2004,

elle est membre de plusieurs groupes de travail et de pilotage sous l'égide

du ministère de la Recherche et, depuis 2012, vice-présidente du GFII.

odille.hologne@ versailles.inra.fir

Des exemples concrets sont identifiables dans chacun des 5 items ci-dessus et décrits dans un numéro spécial du Cahier des Techniques ${ }^{7}$. Toutefois, nous prenons insuffisamment le temps de tirer les leçons de nos expériences et l'apprentissage avec les autres, scientifiques ou partenaires, reste un défi.

Nous avons pu ainsi impliquer les professionnels IST dans la dynamique de la fonction et maintenir leur engagement dans les projets en réinvestissant dans les outils et les compétences grâce à un plan de formation national. Le maintien de la motivation est un challenge permanent.

Notre offre de service est devenue plus visible auprès des scientifiques, notamment grâce à un site intranet qui donne accès aux services en ligne. Les activités de formation sont un véritable vecteur de promotion de nos compétences et services. Ce dispositif d'animation de la fonction IST est devenu un exemple transposé dans d'autres fonctions à l'Inra. •

1 http://referens.univ-poitiers.fr/version/men/liste_emplois.asp?dcp=FF\&corps=\%25\&intitul $=\&$ Submit $2=$ Trouver $+\% 21$

2 0. HOLOGNE, F. BOUDET-BONE, « FuturIST : un projet pour accompagner l'évolution des métiers de l'information scientifique et technique », Cahier des Techniques de I'INRA, 2012, p.5-12. http://prodinra.inra.fr/?locale=fr\#ConsultNotice:178386

3 Themaclic http://wwwl.montpellier.nra.fr/themaclic

4 Veille, bibliométrie, open access, gestion des connaissances, édition, services de proximité, technologies de I'IST, formation, communication.

5 http://fr.wikipedia.org/wiki/Organisation_apprenante

6 http://w3.ualg.pt/ mzacaria/gic/HBR-Building-a-Leaning-Organization.pdf

7 E. MARCHOUX, O. HOLOGNE. L'Information scientifique et technique à I'Inra, des compétences au service de la recherche. Paris, INRA, 2012.

http://prodinra.inra.fr/?locale=fr\#ConsultNotice:179218 
organisation ] Un groupement d'intérêt public : cette forme juridique particulière a accompagné l'histoire d'un réseau et favorisé son évolution. Car au-delà des avantages, somme toute traditionnels, procurés par la mutualisation de produits et services, on passe, avec le GIP, d'un réseau d'individus à un réseau d'établissements, une dimension qui suscite l'émulation, des partenariats et la reconnaissance.
Après avoir été documentaliste au conseil général du Val-de-Marne, puis responsable de la documentation à la chambre régionale des comptes de Rhône-Alpes, Nathalie Berríu est coordinatrice générale du réseau Ascodocpsy depuis juin 2002. Titulaire d'une maîtrise de sociologie politique et d'un DESS documentation et technologies avancées, depuis 2011 elle est certifiée coach et, depuis 2012, certifiée par le CFPJ responsable de communication.

Nathalieberriau@gmail.com

\section{Un GIP pour la gouvernance d'Ascodocpsy}

A scodocpsy ${ }^{1}$, réseau documentaire représentant 91 établissements en santé mentale en France métropolitaine et dans les DOM, regroupe 150 documentalistes qui exercent dans près de 110 centres de documentation et bibliothèques.

Le réseau poursuit quatre objectifs : favoriser la coopération documentaire entre les établissements francophones de santé mentale ; promouvoir le développement des centres de documentation par le recrutement de professionnels, la formation permanente et la création d'un statut de documentaliste; développer des partenariats avec les organismes acteurs dans le champ de la santé mentale et renforcer la diffusion des publications francophones spécialisées en psychiatrie et sciences connexes.

Pour atteindre ces objectifs, une série de produits et services documentaires ${ }^{2}$ classiques ont été créés : un site web $^{3}$, une base bibliographique partagée $^{4}$, un thésaurus ${ }^{5}$, une veille réglementaire, scientifique et éditoriale. Ce qui est moins classique, c'est la forme d'organisation retenue pour administrer le réseau. Depuis 1999, en effet, Ascodocpsy est devenu un groupement d'intérêt public (GIP), soit un établissement public permettant d'associer dans un projet des établissements publics et privés. Le GIP Ascodocpsy a été agréé par les ministères de la Santé et de l'Économie pour une durée de quinze ans.

\section{BREF RETOUR EN ARRIËRE}

Le réseau Ascodocpsy est créé en 1984. À ses débuts, il se réduit à des échanges informels entre quelques documentalistes de centres hospitaliers spécialisés en région. Très vite, le nombre de ses membres augmente tout comme le volume des échanges. Très vite aussi apparaissent une série de produits documentaires (catalogues collectifs, vocabulaire), des procédures communes (formats de saisie communs, bordereaux d'emprunt) et une incitation à s'équiper d'un même logiciel.

Dès 1993, l'idée de structurer le réseau s'impose aux documentalistes comme une nécessité. Avec le développement du réseau, des demandes d'adhésion affluent et des organismes divers ${ }^{6}$ proposent des collaborations. Mais comment répondre à ces demandes sans avoir de mandat ? Comment prendre des décisions vitales si l'on veut qu'un réseau amorcé et qui attire de nouveaux membres poursuive sa croissance ? S'ajoute à cela la volonté de le doter d'une personnalité morale pour asseoir une représentativité émanant des documentalistes du réseau mais aussi des directions et du corps médical ${ }^{7}$.

Jean-Damien Chéné ${ }^{8}$, documentaliste au Centre hospitalier de la Roche-surYon, et Marie-Thérèse Mercier, documentaliste au Centre hospitalier spécialisé de Saint-Egrève, représentant leurs collègues, se rapprochent des directeurs de leurs établissements pour obtenir ce mandat. Après de nombreux échanges qui vont durer près de six ans, ils obtiennent le soutien des deux associations représentatives des directeurs et des psychiatres présidents de commissions médicales d'établissement $(\mathrm{CME})^{9}$. Ensemble, ils transformeront le réseau jusqu'alors informel de professionnels de l'information en un réseau d'établissements.

1 Ascodocpsy, d'abord Association de coopération documentaire en psychiatrie, deviendra Assistance et coopération documentaire en psychiatrie, puis Réseau documentaire en santé mentale.

2 Liste complète : http://www.ascodocpsy.org/ Ascodocpsy-vous-propose-des

3 http://www.ascodocpsy.org

4 SantéPsy, base documentaire accessible en ligne, centralisant les références bibliographiques des unités documentaires des établissements adhérents, signale surtout des documents en langue française en psychiatrie et santé mentale. http://www.ascodocpsy.org/ santepsy/Base/FormulaireRecherche

5 SantéPsy, premier thésaurus en langue française spécialisé en psychiatrie et en santé mentale http://www.ascodocpsy.org/santepsy/Thesaurus/ 6 La Fédération française de psychiatrie, la revue Santé mentale entre autres.

7 Synthèse: « Pour une structuration juridique du réseau » http://www.ascodocpsy.org/IMG/pdf/ structuration_juridique_sept1996-2.pdf 8 Une journée organisée pour les dix ans du GIP leur a rendu un hommage officiel pour leur engagement dans la réussite de ce projet : http://www.ascodocpsy.org /Anniversaire-Ascodocpsy-Informer

9 L’Association des établissements participant au service public de santé mentale (Adesm) et l'Association nationale des présidents et vice-présidents de commissions médicales d'établissements des centres hospitaliers spécialisés (ANPCME). 
L'objectif d'avoir une structure juridique pour pérenniser les outils et productions documentaires créés depuis 1984 est désormais atteint. Une charte est adoptée en 2004. Elle définit les engagements des membres sur le plan éthique et précise les compétences professionnelles attendues, les missions communes et les principes de fonctionnement ${ }^{10}$. Quel bilan tirer treize ans après la création du GIP ?

\section{LES POINTS FORTS}

Le premier bénéfice pour le réseau devenu un GIP est de pouvoir recruter des permanents. Léquipe de coordination $^{11}$, en lien avec le comité technique, assure le développement et la réalisation des objectifs d'Ascodocpsy. Elle prend le relais des documentalistes sur des tâches techniques ou organisationnelles, leur permettant de se concentrer sur les projets qui sont leur cœur de métier tels que l'indexation partagée de documents pour constituer la base documentaire du réseau ou l'élaboration du thésaurus.

L'appui des deux associations fondatrices se révèle être un atout pour obtenir les soutiens de partenaires extérieurs comme les fédérations hospitalières ou le ministère de la Santé. Le déplacement des documentalistes est facilité $^{12}$ par le fait que ce sont des établissements qui sont adhérents et non des individus, et cet engagement institutionnel se traduit par le paiement d'une cotisation annuelle ${ }^{13}$ qui en garantit son fonctionnement.

La représentation tripartite dans les instances par les documentalistes, directeurs et les présidents de CME permet la prise de décisions en s'appuyant sur les compétences professionnelles de ces trois acteurs du réseau, chacun apportant son expertise lors des discussions : les directeurs et médecins ne discutent pas les choix techniques des documentalistes, indiquant par là même la confiance dans leur professionnalisme.

L'accélération du développement des projets est un autre bénéfice. Site web, base documentaire, thésaurus, lettres d'information, partenariats avec les éditeurs de revues ou d'ouvrages en matière d'indexation, organisation de journées d'étude, agrément comme organisme de formation, partenariats avec des fédérations professionnelles, etc. sont des exemples de réalisations de ces dix dernières années.

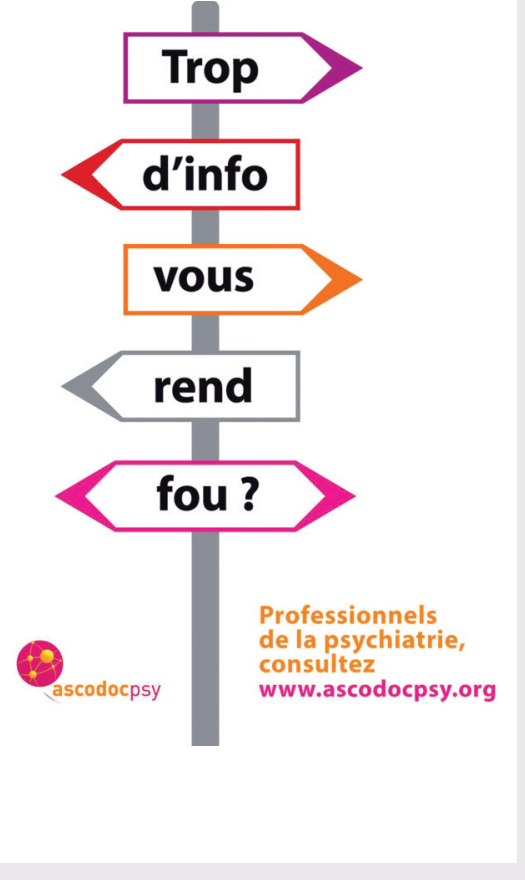

La rédaction de plans de communication et un travail en profondeur sur les valeurs à partager au sein du réseau participent à l'amélioration de son fonctionnement ${ }^{14}$. Ascodocpsy, engagé dans une démarche de communication, produit des cartes publicitaires aux slogans décalés («Trop d'info vous rend fou? » ou encore « Chercher l'info vous déprime? »). Une commission d'archivistes hospitaliers, agrégée au réseau des documentalistes peu après la création du GIP, a produit des guides remarqués ${ }^{15}$ par la communauté archivistique hospitalière et les Archives de France.

\section{MARGES DE PROGRESSION}

En contrepartie de la légitimité, le réseau a hérité des lourdeurs administratives. Le circuit de décision s'est allongé du fait du nombre des interlocuteurs officiels qui interviennent dans le processus. Certaines décisions prises par les instances apparaissent comme déconnectées de la réalité du terrain.

Quant à la représentation tripartite, si elle figure dans les textes, elle ne se traduit pas forcément sur le terrain : l'assemblée générale où sont invités les trois représentants de l'établissement est surtout fréquentée par les seuls documentalistes.

\section{STOP OU ENCORE?}

«Ascodoc un jour, Ascodoc toujours ", ce sont sur ces mots que terminait son mandat le précédent président d'Ascodocpsy. En décembre 2012, lors d'une réunion commune des instances organisée sur l'après 2015, date de la fin de l'agrément ministériel attribué au réseau Ascodocpsy en tant que GIP, c'est sans hésiter que les représentants du réseau ont répondu « Oui » à la question posée de la poursuite de l'aventure du GIP. •

\section{Instances, commission et coordination dans le GIP}

Un groupement d'intérêt public (GIP)

fonctionne comme une association loi

1901. Une assemblée générale élit un

conseil d'administration (CA), les administrateurs élisent parmi eux un bureau qui met en oeuvre les décisions du CA, et un conseil scientifique accompagne les instances par ses réflexions sur la politique mise en œuvre par le réseau. Trois représentants de chaque établissement adhérent votent lors de l'assemblée générale : le documentaliste, le directeur et le psychiatre président de la commission médicale d'établissement. Cette représentation tripartite garantit les liens du partenariat institutionnel nécessaire au développement de la fonction documentaire au sein des établissements.

Le comité technique, composé des responsables des treize commissions ${ }^{16}$ et de trois documentalistes, a pour mission de superviser, valider et assurer la mise en œuvre des travaux du réseau en collaboration avec l'équipe de coordination. •

$10 \mathrm{http}: / /$ www.ascodocpsy.org/Archives-du-GIP 11 http://www.ascodocpsy.org/Equipe-de-coordination. 12 Pour des raisons budgétaires, certains établissements sont plus réticents que d’autres à autoriser les déplacements de leurs agents.

13 Montant des cotisations annuelles http://www.ascodocpsy.org/Nous-rejoindre-adhererau-reseau

14 Le second plan de communication s'appuie sur les travaux des étudiants de masterl de l'université Jean Moulin Lyon 3.

15 Rubrique commission archives hospitalières http://www.ascodocpsy.org/-Ressources-documentaires15 Les commissions

http://www.ascodocpsy.org/-Groupes-de-travail- 
[ analyse ] L'organisation en réseau, c'est une histoire bibliothèque de la Maison de l'Orient et de la Méditerranée ancienne pour le CNRS qui a une culture réseau bien à Lyon. Elle représente le réseau régional Isidora au comité établie. De cette présentation, on retiendra les points de pilotage de Renatis.

forts de ce mode d'organisation : une meilleure remontée des problèmes, une réelle adaptation aux besoins, une réflexion collective et une vision prospective pour le plus grand bénéfice de la recherche.

\section{Les réseaux métiers en IST au CNRS}

Le Centre national de la recherche scientifique (CNRS) a créé en 2000 une mission pour les ressources et compétences technologiques $(\mathrm{MRCT})^{1}$ dont l'objectif est d'initier, structurer et animer des communautés partageant une technologie (plasmas froids, laser femto, haute pression, microscopie, etc.) ou un ensemble de métiers (mécanique, électronique, information scientifique, etc.).

\section{Cinq réseaux en région et un réseau national}

Dès 2001, cette mission soutenait la création d'Isidora $^{2}$, premier réseau régional en IST qui réunit les documentalistes de la région Rhône-AlpesAuvergne, puis de Go'Doc ${ }^{3}$ pour les professionnels d'Ile-de-France. Entre 2003 et 2006, elle favorise l'émergence de trois autres réseaux : Arpist ${ }^{4}$ (Aquitaine), Mistral-Doc ${ }^{5}$ (Provence et Côte d'Azur), Doccitanist $^{6}$ (Midi Pyrénées-Languedoc Roussillon).

Constitués à l'initiative de quelques documentalistes, ces réseaux rassemblent des professionnels de l'IST exerçant dans des unités CNRS, quelles que soient les disciplines. La grande diversité des situations des personnels - travail en équipe ou isolés, des statuts (personnel CNRS ou universitaire) et des formations - expliquent le besoin de se regrouper.

Ces réseaux régionaux, fondés sur le volontariat, se sont organisés de façon similaire : un comité de pilotage, une liste de diffusion, des groupes de travail chargés de l'animation du réseau (création du site web, organisation de formations) ou centrés sur des problématiques (enquête métiers, droit d'auteur, etc.). Les crédits, alloués par la MRCT et gérés par des délégations régionales du CNRS, financent les réunions des comités de pilotage et des groupes de travail et l'organisation de journées thématiques pour les membres d'un réseau.

En 2006, la MRCT approuve la création de Renatis ${ }^{7}$, réseau national dont l'objectif est de mutualiser certaines actions menées au niveau régional, de fédérer les membres des réseaux régionaux et les professionnels dans des régions non organisées en réseau et, surtout, d'être un interlocuteur pour les instances nationales.

\section{Pilotage des réseaux}

Le mode de fonctionnement de Renatis est similaire à celui des régions. Son comité de pilotage, constitué de représentants des réseaux régionaux et de membres nommés par le comité après examen d'une candidature, a créé des groupes projets qui travaillent sous sa responsabilité. Le groupe chargé de la formation a un fonctionnement particulier du fait de sa vocation à être pérennisé, au moins pour l'organisation tous les 2 ans d'une formation nationale baptisée FRéDoc (Formation des réseaux de la documentation).

Les demandes annuelles budgétaires sont centralisées par Renatis qui les adresse à la MRCT. Celle-ci partage le budget global qui lui est attribué avec les réseaux régionaux et conserve une enveloppe pour son propre fonctionnement. Les crédits distribués sont gérés dans les délégations régionales du CNRS, chaque réseau étant autonome pour leur utilisation.

Les échelles régionale et nationale sont complémentaires: l'une correspond à une logique de proximité concrétisée par des journées thématiques et des formations en région, l'autre concourt à améliorer la visibilité des professionnels de l'IST en étant un interlocuteur pour les instances nationales et en menant des actions de plus grande envergure. Renatis, correspondant pour la Direction de l'information scientifique et technique du CNRS (DIST ${ }^{8}$ ), participe ainsi aux réflexions menées dans le cadre de la Bibliothèque scientifique nationale $\left(\mathrm{BSN}^{9}\right)$. 


\section{Autonomie des réseaux : un fonctionnement indépendant des laboratoires}

Ce fonctionnement, indépendant des laboratoires de recherche et des instituts du CNRS et autonome financièrement, est à la fois un atout et une faiblesse des réseaux métiers.

Un atout pour les personnels qui qui peuvent mener une réflexion prospective et axer le travail du réseau sur des problématiques dont l'application n'est pas immédiate pour les tâches quotidiennes du laboratoire et qui ne s'inscrit pas dans des projets en cours. Cette prospective, indispensable, permet aux personnels d'appréhender les enjeux liés aux développements technologiques et aux évolutions institutionnelles et de se former à ces nouveaux domaines. Le paysage documentaire des personnels IST est en pleine mutation, les frontières entre la documentation dans les laboratoires et la documentation à l'université sont de plus en plus floues et la mutualisation s'impose partout. Seul le travail en réseau permet de conduire une réflexion indépendamment de l'expérience individuelle de chacun.

Contrairement aux actions menées dans les réseaux thématiques dépendants des instituts dont le fonctionnement et les orientations sont partagés entre chercheurs et professionnels de l'IST au bénéfice direct des chercheurs, l'intérêt du travail en réseau transdisciplinaire, et son axe principal, centré sur les aspects métiers de l'info-doc, n'est pas immédiatement perceptible par les chercheurs et, en particulier, par les directeurs de laboratoire, qui seuls peuvent autoriser les personnels placés sous leur responsabilité à s'investir dans les réseaux.

En revanche, le fait que le laboratoire de recherche ne finance ni les frais de mission de son personnel pour participer aux groupes de travail, ni les frais des formations organisées par le réseau (pris en charge par les formations permanentes du CNRS en région), facilite l'autorisation et le soutien du directeur du laboratoire à son personnel. Si le manque de retombées immédiates pour le laboratoire est un frein au travail dans les réseaux, l'absence de financement direct favorise l'implication des personnels.

Par ailleurs, l'adhésion au réseau et la participation aux groupes projets se fait sur la base du volontariat et, même face à une direction de laboratoire consciente de l'intérêt du travail effectué, le personnel ne bénéficie d'aucune décharge face au travail quotidien, ce qui fragilise et pénalise l'activité des groupes de travail. Les réflexions et actions menées permettent pourtant d'améliorer les services offerts aux chercheurs par une élévation du niveau global des compétences des personnels IST, en matière de choix technologiques par exemple, qui profite aux laboratoires et au CNRS dans son ensemble. Mais la difficulté de mesurer l'impact de ce travail dans le laboratoire, couplé au mode autonome de fonctionnement du réseau, peine à donner une visibilité aux actions menées et à leur finalité.
Même si s'investir dans le travail au sein des réseaux n'est pas toujours simple, le bilan d'ensemble est très positif : d'une part, le mode d'animation et de pilotage participatif qui permet de faire remonter directement les problèmes de terrain et de les résoudre est un mode bottom up motivant pour les personnels ; d'autre part, ce sont les professionnels en exercice dans les laboratoires qui pilotent ces réseaux et qui adaptent donc les formations aux besoins des personnels. Les réflexions collectives, enfin, permettent de construire une vision prospective du métier. •

\section{LES RÉSEAUX EN IST AU CNRS}

À côté des réseaux technologiques métiers tel Renatis, de nombreux réseaux documentaires thématiques ne sont pas placés sous la tutelle de la MRCT mais liés aux instituts scientifiques du CNRS :

- Démocrite : portail de ressources documentaires de l'IN2P3 (physique nucléaire et physique des particules) ${ }^{10}$

- Doc'PI@nets : réseau des professionnels de la documentation de I'Institut des sciences de l'univers ${ }^{11}$

- Rédovie : réseau des documentalistes en sciences de la vie ${ }^{12}$

- Doc-ST21 : réseau des documentalistes en sciences et technologies de l'information et de la communication et sciences pour l'ingénieur ${ }^{13}$

- DocAsie : réseau de documentalistes et bibliothécaires travaillant sur l'Asie $^{14}$

- Isore : réseau en sciences humaines et sociales ${ }^{15}$.

Ces réseaux, même soutenus par les Instituts, ne sont pas dotés

d'une structure propre, mais disposent de comités de pilotage pour coordonner leurs actions, de listes de diffusion pour communiquer et de groupes de travail.

Deux de ces réseaux thématiques sont organisés en groupement de services (GDS), structures CNRS dotées d'une direction et d'un budget propres : le RNBM (réseau national des bibliothèques de mathématiques) ${ }^{16}$ et Frantiq (fédération et ressources sur l'Antiquité) ${ }^{17}$.

L'Institut des sciences humaines et sociales, qui comporte le plus grand nombre de professionnels et dont l'implantation dans les laboratoires est très forte, a créé un réseau de correspondants pour l'information scientifique et technique, dont le rôle est, outre le partage de compétences et de savoir-faire, d'assurer le relais entre l'Institut et les laboratoires. • |||||||||||||||||||||||||||||||||||||||||||||||||||||||||||||||||||||||||||||||||||||||||||||||||||||||||||||||||||!||

\author{
1 http://www.mrct.cnrs.fr \\ 2 http://isidora.cnrs.fr \\ 3 http://www.godoc.u-psud.fr \\ 4 http://www.arpist.cnrs.fr \\ 5 http://mistral.cnrs.fr \\ $6 \mathrm{http}: / /$ doccitanist.lirmm.fr \\ $7 \mathrm{http}: / /$ renatis.cnrs.fr \\ 8 http://www.cnrs.fr/dist. En juillet 2010, le CNRS s'est doté d'une Direction dédiée à \\ l'information scientifique et technique (DIST), lieu d'élaboration de la politique et de \\ pilotage des activités du CNRS en matière d'IST. \\ 9 La Bibliothèque scientifique nationale (BSN) fait partie des Très grandes infrastructures \\ de recherche (TGIR) portées par le ministère de l'Enseignement Supérieur et de la \\ Recherche (MESR). Renatis participe aux groupes de travail 8 (Fourniture de documents, \\ prêt) et 9 (Formations, compétences, usages). \\ $10 \mathrm{http}: / /$ documentalistes.in2p3.fr \\ $11 \mathrm{http}: / /$ bibliocnrs.netvibesbusiness.com/docplanets \\ 12 http://redovie.cnrs.fr \\ $13 \mathrm{http}: / /$ doc-stzi.cnrs.fr \\ $14 \mathrm{http}$ ://docasie.ish-Iyon.cnrs.fr \\ 15 http://www.isore.cnrs.fr \\ $16 \mathrm{http}: / /$ www.rnbm.org \\ $17 \mathrm{http}: / / w w w . f r a n t i q . f r$
}


L'Aciége dont, les écoles sont de plus en plus internationales, est en relation régulière avec des associations regroupant de la même façon des responsables de centres de documentation des meilleures Business Schools : l'EBSLG en Europe, l'ABLD aux États-Unis et l'APBSLG ${ }^{7}$ en Asie. L'échange d'informations et de bonnes pratiques est une importante fonction de ces relations.

La grande force de l'association est le travail en réseau, la mutualisation des savoirs et savoir-faire. La communication informelle entre ses membres est un outil d'information sans prix, renforçant l'implication de chacun et favorisant la résolution de problèmes.

\section{Concurrence}

Dans les années 2010, les écoles de commerce font face à une concurrence exacerbée en raison :

- des progrès technologiques et de la compétition dans le monde des entreprises qui conduit à une plus grande segmentation des besoins et à une demande plus spécialisée (finance, marketing, RH, etc.). Les écoles qui n'atteignent pas la taille critique pour couvrir tous ces domaines doivent faire des choix ;

- de la globalisation qui impose de nouveaux challenges: les écoles doivent recruter un corps professoral international sur un marché de chercheurs et d'enseignants qui ressemble à celui des sportifs de haut niveau.

C'est dans ce contexte que cinq écoles de commerce

fusionnant, pour constitue Sigle du FBS la France Business Schools $(\mathrm{FBS})^{8}$. Ces écoles sont représentées dans l'Aciége par les responsables des centres de documentation qui se connaissent déjà bien grâce à ce réseau.

\section{Mutualisation}

En septembre 2012, la direction de la France Business School ne souhaitait avoir pour la documentation qu'un seul interlocuteur. Les cinq responsables ont alors pris l'initiative de lui soumettre un CV collectif mettant en avant leurs compétences communes (adaptation au travail en équipe qu'elles pratiquent au sein de l'Aciége, responsabilité d'un centre documentation) et leur complémentarité. Sur le slogan de l'Ecole: « New World. New School», elles ont proposé une solution innovante, en s'inspirant du modèle anglo-saxon : Le FBS Library Committee, nouvelle structure fondée sur un nouveau mode d'organisation.

En février 2013, la direction a validé le Library Committee et proposé un nouvel organigramme composé d'une part des cinq responsables Co-Workingspaces à Amiens, Brest, Clermont, Poitiers et Tours, rattachées hiérarchiquement au Directeur du Learning Hub, et d'autre part d'un professeur et d'un étudiant, relais du Learning Hub sur chacun des campus.

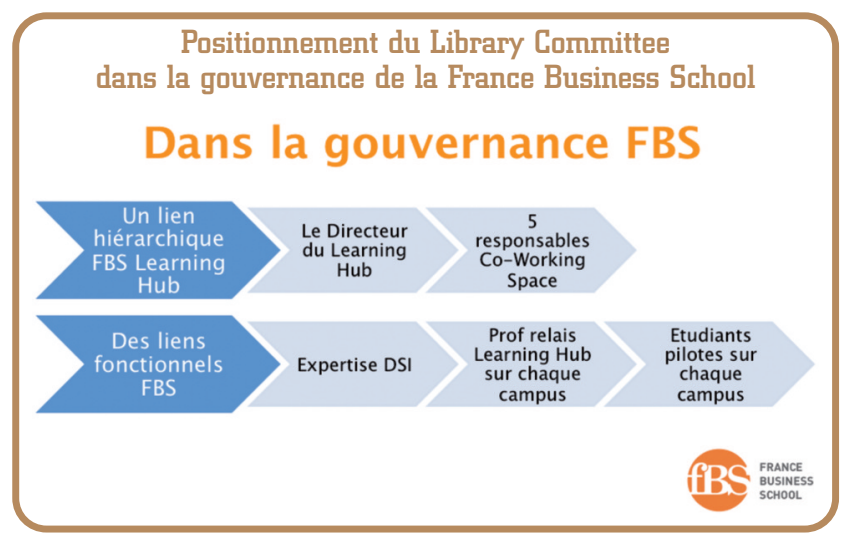

Le Library Committee aura pour objectif de :

- définir et mettre en place une politique documentaire multicampus ;

- coordonner les activités, produits et services ;

- mutualiser les ressources et les outils de recherche ;

- contribuer au développement du Learning Hub.

Il comprendra un forum de discussion et sera fondé sur la transversalité, intégrant membres du corps professoral et étudiants représentants de la communauté FBS. Il nécessitera l'implication des équipes respectives sur les différents chantiers. Les responsables des centres de documentation et le directeur du Learning Hub auront voie délibérative. La validation finale sera faite par le directeur du Learning Hub. Le comité se réunira 1 à 2 fois par mois à distance via des outils collaboratifs et en séance plénière 1 à 2 fois par an au siège parisien de la FBS.

Cette initiative innovante en terme d'organisation montre combien la force d'un réseau professionnel est importante et qu'il peut influencer de façon radicale les décisions d'une direction d'établissement.

Un article récent du Bulletin des bibliothèques de France (BBF) titrait: « Bibliothèques universitaires et concurrence ». Il rappelle que les universités françaises aussi « vont rentrer en concurrence et, avec elles, l'ensemble de leurs services formations, recherchemais aussi BU [...]. Elles vont devoir prouver leur efficacité, non seulement par l'accomplissement de leurs missions traditionnelles mais également en s'inscrivant pleinement dans la stratégie de développement et d’image de leur tutelle $»^{9}$. •

$1 \mathrm{http}: / /$ www.esdes.fr/programmes/palmares-des-ecoles-de-commerce-les-limitesdu-consumerisme--81923.kjsp?RH=1184748564632

2 http://www.mba50.com/fifteen-years-of-influence-the-ft-mba-ranking-1999-2013 3 Association to Advance College School of Business http://www.aacsb.edu/; Association of Mbas http://www.mbaworld.com/;

Equis http://www.efmd.org/accreditation-main/equis

$4 \mathrm{http}: / /$ www.cge.asso.fr/qui-sommes-nous/commissions/10-chapitre-des-ecoles-demanagement

5 http://ww.aciege.org

$6 \mathrm{http}: / /$ www.aciege.org/index.php?option=com_content\&view=article\&id=116:

thesaurus-du-management\&catid=40:publications

7 European Business Schools Librarians' Group http://www.ebslg.org/;

Academic Business Library Directors http://www.abld.org ;

Asia Pacific Business School Librarian's Group http://www.isb.edu/apbslg/ 8 http://www.france-bs.com/?gclid=CK3×3uuLsrYCFXDKtAodJWwAEQ

9 V. APPEL, L. LACOTE-GABRYSIAK. « Bibliothèques universitaires et concurrence ou comment la communication devrait venir aux bibliothèques ». Bulletin des bibliothèques de France, $n^{\circ} 4$, p. 44-48 